\title{
SOLIDIFICATION/STABILISATION DES VASES À BASE DE CHAUX ET ADDITIFS CONCEPTS COMPORTEMENTAUX ET LIXIVIATION
}

\author{
Francis Rey* et**, Daniel Levacher*, Jean-Louis Quénec'h** \\ upres A 6/43 Caen - École supérieure d'ingénieurs des travaux de la construction de Caen
}

\begin{abstract}
Devant la situation actuelle préoccupante du devenir des matériaux vasards contaminés, le traitement par Solidification/Stabilisation (S/S) est une solution pouvant permettre leur valorisation en génie civil.

Après une étude qui a permis d'identifier les mécanismes physico-chimiques clefs responsables des évolutions physico-mécaniques complexes de ce type de composites, une étude de lixiviation a été conduite afin d'étudier l'efficacité et le type d'immobilisation des métaux lourds au sein des matrices. Les résultats nous amènent à considérer différents potentiels d'immobilisation suivant les métaux considérés.
\end{abstract}

Sludge materials pose a problem for harbour and coastal engineers in respect with the environment rules. The stabilisation/solidification (S/S concept) constitutes one way to reuse them as civil construction materials. After détermination the fundamental mechanisms governing the complex physical-chemical evolutions of this composites, lixiviation tests were improved in order to study the efficiency and type of contaminants mobility. The results allow to consider different mobility potentials according to the studied metals.

\section{INTRODUCTION}

Les activités de dragage génèrent annuellement des volumes de matériaux vasards considérables (environ 50 millions de $^{3}$ en France; Alzieu 1999). Les gestions traditionnelles de ces matériaux semblent condamnées à plus ou moins court terme du fait de la raréfaction des sites de dépôts terrestres et suite à l'évolution de la réglementation pour les immersions en mer (Géode 1994). En effet, une importante part de ces matériaux contient des quantités non négligeables de contaminants, ce qui représente des risques pour le milieu marin. Devant cette situation la valorisation après traitement de $S / S$ est une solution alternative attrayante. Plusieurs études ainsi que des projets industriels, notamment au Japon (Kamon et al. 1990) et au Canada (Stegeman 199I) semblent confirmer l'intérêt de la technique.
Dans ce cadre, des travaux visant à définir les propriétés mécaniques, physiques et environnementales des matériaux après une technique de solidification/stabilisation ont été menés. Parmi ceux-ci, le traitement des vases à la chaux plus additifs est une approche intéressante.

L'ajout de chaux vive à la vase brute et remaniée en sortie du dragage, confère rapidement aux vases une structure grumeleuse organo-minérale sitôt que la réaction fortement exothermique d'extinction a eu lieu. Les opérations de manipulation, stockage ou transport, sont alors simplifiées. À ce stade, l'obtention d'un matériau monolithique, susceptible de répondre aux exigences mécaniques et environnementales liées, par exemple, à une utilisation en construction routière, passe par l'ajout d'un liant et un compactage. Cette méthode permet de constituer un matériau monolithique, insoluble et le moins poreux possible.

\section{CARACTÉRISTIQUES ET POLLUTIONS DES VASES UTILISÉES}

Les vases utilisées proviennent du port autonome du Havre (Seine-Maritime) et du port de plaisance de Dives-sur-Mer (Calvados). Les vases du Havre sont issues d'un dragage mécanique à godets alors que la vase de Dives provient d'un dragage hydraulique. In situ, les matériaux ont une teneur en eau (masse d'eau/masse sèche) variant ordinairement de 130 à $160 \%$. Cette variation est essentiellement due à l'état de consolidation de la vase alors que le moyen de prélèvement aura une influence considérable dans un second temps. Les principales caractéristiques physico-chimiques des prélèvements sont résumées dans le tableau I.

Les deux vases sont semblables d'un point de vue granulométrique et minéralogique. Les phases majoritaires sont le quartz et les carbonates. Dans les deux cas également, les minéraux phyliteux sont peu présents. Ces caractéristiques sont une spécificité des sédiments côtiers de la baie de Seine. La solution interstitielle contient des sels dissous provenant de la nature marine des matériaux. La valeur du carbone organique total (COT) suffit à donner un caractère nettement organique au produit en constituant un gel organométallique noir-brun caractéristique des matériaux vasards et de leurs propriétés particulières de consolidation et de 
viscosité. La matière organique est principalement constituée de substances humifiées $(\mathrm{SH})$ dont les constituants sont l'humine (insoluble et fortement associée à la phase minérale), les acides humiques (solubles en milieu alcalin) et les acides fulviques en solution. Ces composés, qui sont des polymères complexes de structures non définies à noyaux aromatiques, ont de fortes propriétés de complexation avec les éléments et particules chargées (Busnot 1991). Ils constituent de ce fait les principaux agents de concentration et de rétention des contaminants au sein du matériau vasard après floculation/coagulation et décantation des agrégats minéralo-organiques.

\section{Tableau I : Caractéristiques des vases utilisées}

\begin{tabular}{|c|c|c|}
\hline Caractéristiques physico-chimiques & Dives & Le Havre \\
\hline Teneur en eau : w (\%)* & $170-182$ & $128-134$ \\
\hline Densité de la phase solide : $\mathrm{d}^{*}$ & 2,65 & 2,63 \\
\hline Indice de plasticité : $I_{p}(\%)^{*}$ & 5 & 55 \\
\hline Argile $(<$ à $2 \mu \mathrm{m})(\%)^{* *}$ & 23 & 31 \\
\hline Silt $(2$ à $64 \mu \mathrm{m})(\%) * *$ & 73 & 67 \\
\hline Sable (> à $64 \mu \mathrm{m})(\%)^{* *}$ & 4 & 2 \\
\hline Indice de floculation : If (\%)*** & 60 & 46 \\
\hline Quartz (\%) & 40 & 43 \\
\hline Carbonates (\%) & 42 & 38 \\
\hline Feldspaths et micas (\%) & 5,5 & 3 \\
\hline Argiles (\%) & 3 & 3 \\
\hline Chlorures (\%) & 3,08 & 2,40 \\
\hline Sulfates (\%) & 0,41 & 0,17 \\
\hline COT (\%) & 2,88 & 3,23 \\
\hline \multicolumn{3}{|c|}{$\begin{array}{l}\text { * Pour un séchage à } 105^{\circ} \mathrm{C} \\
\text { ** Matériau défloculé }\left(\mathrm{H}_{2} \mathrm{O}_{2} \text { et hexamétaphosphate de sodium) }\right. \\
\left.\text { *** } I_{\mathrm{f}}=100 \text {. ( } \mathrm{d}_{50} \text {, vase brute }-\mathrm{d}_{50} \text {, vase déloculée }\right) / \mathrm{d}_{50} \text {, vase brute }\end{array}$} \\
\hline
\end{tabular}

Tableau 2 : Teneurs en métaux lourds des vases

\begin{tabular}{lrrrrrr} 
Métaux* (mg/kg sec) $\mathbf{C d}$ & $\mathbf{C r}$ & $\mathbf{C u}$ & $\mathbf{N i}$ & $\mathbf{P b}$ & $\mathbf{Z n}$ \\
\hline Dives & 0,72 & 75 & 35 & 23 & 57 & 195 \\
\hline Le Havre & 1,64 & 109 & 56 & 23 & 102 & 209 \\
\hline BdF** & 0,5 & 45 & 35 & 20 & 47 & 115 \\
Niveau I** & 1,2 & 90 & 45 & 37 & 100 & 276
\end{tabular}

* : As, $\mathrm{Hg}$, Se et PCB : polluants non-dosés

* : Recommandation Géode 1994

La teneur totale des contaminants métalliques est obtenue après minéralisation totale de l'échantillon conformément à la norme NF X $3|-| 5 \mid$. Le dosage est ensuite réalisé après reprise des cendres par spectrométrie d'absorption atomique four. Les résultats sont présentés dans le tableau 2 .
D’après la recommandation Géode (1994), la vase de Dives, pour ces métaux, est faiblement nuisible pour l'environnement marin dans la mesure où la contamination est comprise entre le bruit de fond « géologique » (BdF) et le niveau I de cette grille. Pour la vase du Havre, la situation est bien différente. Les teneurs en $\mathrm{Cd}, \mathrm{Cr}$, $\mathrm{Cu}$ et $\mathrm{Pb}$ sont comparativement bien supérieures à la vase de Dives tout en étant supérieures au niveau I. Dans ce cas, l'immersion comporte un risque, d'autant plus que le cadmium (Cd) qui est particulièrement toxique, semble présent à une teneur supérieure à 3 fois le BdF. Pour ce site, trouver une solution alternative à l'immersion est donc une priorité. Le sédiment étudié est, de plus, plutôt représentatif de la moyenne des vases draguées et ne constitue en rien un échantillon particulièrement pollué.

\section{PARAMÈTRES DU PROCÉDÉ DE S/S ET CONFECTION DES ÉPROUVETTES}

Pour cette étude, deux types d'ajouts ont été employés : un ciment CPJ CEM II A/32,5 R courant et des cendres volantes (CV) de houille de centrale thermique.

Avec ces deux types d'ajouts, employés séparément, il a été possible d'apprécier l'effet distinct des deux familles de liants courants appliqués à la solidification/stabilisation. Le CPJ est un liant hydraulique à prise rapide où la formation des composés cimentiers dépend de l'hydratation des éléments anhydres du clinker, alors que la formation des composés pouzzolaniques semblables dépend initialement de la lente dissolution et diffusion interstitielle des phases plus ou moins amorphes de l'ajout.

Les travaux de Boutouil (1998) sur la solidification/stabilisation des vases à base de ciment et additifs ont montré que le type et la classe de ciment (CPJ 32,5; CPA 52,5; CPA PM 52,5), à dosages modérés, avaient peu d'influence sur la résistance des composites vase-ciment. Le choix s'est donc porté sur le plus économique. Les cendres volantes retenues se caractérisent essentiellement dans leur composition par l'absence de chaux. Ainsi, en ajout au mélange vase-chaux, l'effet pouzzolanique attendu sera catalysé par la chaux qui est déjà, dans le mélange initial, théoriquement en excès. Le malaxage des constituants est réalisé en deux fois afin de bénéficier au maximum des propriétés d'absorption et d'évaporation d'eau de la chaux ainsi que de la floculation organo-calcique qui transforme radicalement les boues et minimise les interférences $\mathrm{SH}$-liant. Le mélange de la vase avec la chaux précède donc ( $1 / 2$ journée) celui réalisé avec l'ajout. Le dosage en chaux $(x)$ est un rapport pondéral par rapport à la masse de vase brute. Le dosage en ajout (y) est exprimé par rapport à la masse de mélange vase-chaux après maturation (voir tableau 3).

Immédiatement après le malaxage avec l'ajout, des éprouvettes sont réalisées par compactage quasi statique dans des moules cylindriques suivant un mode opératoire analogue à celui de la norme NF P 98-230-2 sur les sols traités. Les éprouvettes, de $38,6 \pm 0, \mathrm{I}) \mathrm{mm}$ de diamètre sur $77 \pm \mathrm{I}$ ) $\mathrm{mm}$ de hauteur ont, après extrusion, une masse volumique équivalente \pm | \%) à 
Tableau 3 : Dosages et facteurs de changement de volume (FCV) du procédé de S/S

\begin{tabular}{|c|c|c|c|c|c|}
\hline Vase & $\begin{array}{l}\text { Chaux } \\
x(\%)\end{array}$ & $\begin{array}{l}\text { Ajout } \\
y(\%)\end{array}$ & $\begin{array}{l}\text { FCV* } \\
\text { Ciment }\end{array}$ & $\begin{array}{l}\text { FCV* } \\
\text { CV }\end{array}$ & Légendes des figures \\
\hline Dives & $49-52$ & 7 & 1,09 & 1,15 & D-52-7-CV \\
\hline Dives & $49-52$ & 17 & 1,15 & 1,28 & D-52-17-C D-52-17-CV \\
\hline Dives & $49-52$ & 29 & 1,28 & 1,37 & D-52-29-C D-52-29-CV \\
\hline Dives & 42 & 18 & 1,12 & 1,18 & D-42-18-C D-42-18-CV \\
\hline Dives & 36 & 27 & 1,14 & 1,24 & D-36-27-C D-36-27-CV \\
\hline Le Havre & $38-40$ & 7 & 1,07 & 1,10 & $\mathrm{H}-39-7-\mathrm{C} \quad \mathrm{H}-39-7-\mathrm{CV}$ \\
\hline Le Havre & $38-40$ & 17 & 1,12 & 1,19 & $\mathrm{H}-39-17-\mathrm{C} \quad \mathrm{H}-39-17-\mathrm{CV}$ \\
\hline Le Havre & $38-40$ & 29 & $\mid, 21$ & 1,33 & $\mathrm{H}-39-29-\mathrm{C} \quad \mathrm{H}-39-29-\mathrm{CV}$ \\
\hline Le Havre & 33 & 18 & 1,13 & 1,20 & $\mathrm{H}-33-18-\mathrm{C} \quad \mathrm{H}-33-18-\mathrm{CV}$ \\
\hline Le Havre & 27 & 27 & 1,17 & 1,26 & H-27-27-C $\mathrm{H}-27-27-\mathrm{Cl}$ \\
\hline
\end{tabular}

celle obtenue préalablement après un essai Proctor modifié (NF P 94-093). Ainsi les caractéristiques mécaniques et physico-chimiques des éprouvettes sont déterminées pour une consistance correspondant à une énergie de compactage constante et faisant référence dans les travaux routiers. Notons également que les dosages minimaux employés, et particulièrement celui en chaux, sont une conséquence directe de cette méthodologie de confection et notamment de la faisabilité de réalisation de l'essai de compactage dynamique Proctor. Les éprouvettes sont ensuite conservées en moules quasi hermétiques à $20( \pm 2){ }^{\circ} \mathrm{C}$.

Les valeurs du FCV montrent systématiquement, qu'à dosages identiques, les composites avec ajout de CV sont moins compressibles que ceux obtenus avec un ajout de type ciment. Ceci est la conséquence de la densité absolue plus élevée du ciment et de son hydratation avant compactage alors que les $\mathrm{CV}$ sont à considérer à très court terme comme une poudre inerte.

\section{PROBLÉMATIQUE DU TRAITEMENT ET CONCEPTS PHYSICO-MÉCANIQUES}

\section{Interférences des $\mathrm{SH}$ sur les cinétiques de formation des composés de cimentation}

Lors du traitement des vases avec les liants hydrauliques, les substances humiques $(\mathrm{SH})$ interfèrent fortement sur les processus d'hydratation ou de formation des phases cimentières. Les composés organiques agissent comme des agents retardateurs de prise : adsorption sur les surfaces anhydres, complexation avec les ions en solution (Jolicoeur et al. 1998). Ils agissent également ici par immobilisation physiquement des ions dans le gel organique. Ces interférences sont très importantes et font considérablement chuter la résistance des matériaux à court et moyen terme (Kujala et al. 1996). L'action du dosage préliminaire en chaux joue un rôle déterminant dans la cinétique de ce processus retardateur (voir figure I : Rey 1999).

Chimiquement la forte concentration en $\mathrm{Ca}^{2+}$, conduit à
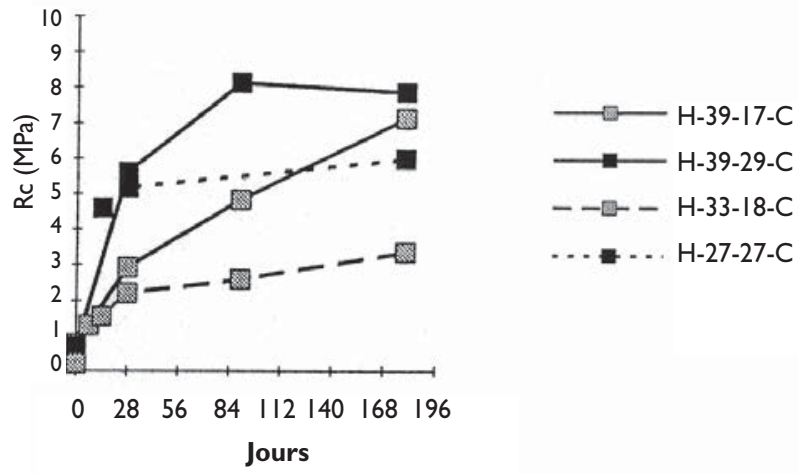

Figure Ia : Composites ciment
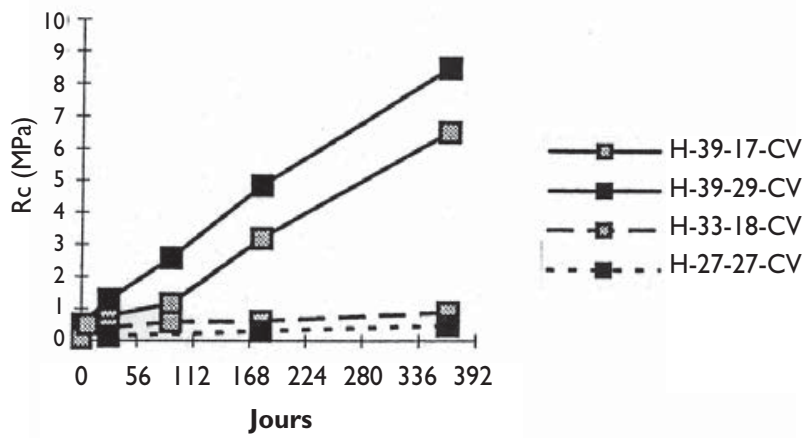

Figure Ib : Composites CV

Figure I : Évolution des résistances en compression simple Influence du dosage en chaux et de la cinétique de réaction

saturer la capacité d'échange cationique des SH. Les futures interférences sur les liants sont ainsi réduites. La chaux est également susceptible de développer une réaction pouzzolanique avec les minéraux naturels résultant de la mise en solution de fines particules minérales peu ou non cristallisées suite à l'attaque de la solution calcobasique (Le Roux 1969). Ceci conduit normalement, à plus ou moins long terme, à la formation, entre autres, de silicates de calcium hydratés $(\mathrm{CSH})$ stables semblables à ceux formés par les ciments et aboutit donc à une solidification. Cependant à court et moyen terme le caractère nettement organique des vases inhibe totalement ce type de réaction (Rey et al. 1998). Outre les effets physico-chimiques inhibiteurs précédemment cités, il faut ajouter le rôle de membrane protectrice que constitue l'humine autour des minéraux.

Physiquement, le dosage en chaux est la cause de la floculation immédiate d'agrégats structuraux minéralo-organiques par complexation multiples $\mathrm{SH}-\mathrm{Ca}-\mathrm{SH}$. Ceux-ci viennent initialement jouer le rôle de « granulats visco-élasto-plastiques» dans les composites alors que les flocons formés sont d'autant plus denses que le milieu est asséché par la formation de portlandite $(\mathrm{CH})$.

\section{Minéralisation/transformation graduelle des SH}


La solution calcobasique des matrices conduit à l'oxydation des $\mathrm{SH}$ ce qui entraîne leur solubilisation/minéralisation progressive. Cette transformation a de nombreuses conséquences et entre autres :

- une libération des éléments complexés ( $\mathrm{Ca}, \mathrm{Al}, \mathrm{Fe} . .$.$) ainsi$ que des polluants;

- une disponibilité des anions constitutifs des matières organiques $\left(\mathrm{CO}_{3}{ }^{2-} ; \mathrm{SO}_{4}{ }^{2-} \ldots\right)$;

- une déstructuration des agrégats minéralo-organiques formés initialement.

Physiquement, la minéralisation/transformation des $\mathrm{SH}$ se traduit par des pertes plus ou moins importantes de matières sèches (Rey 1999).

Ainsi, peu à peu, l'activité chimique pour la formation des $\mathrm{CSH}$ peut se développer dans le cas où celle-ci serait initialement entravée. Ceci conduit alors à une solidification efficace du matériau : insolubilité dans l'eau et résistance de plusieurs MPa. Ce matériau devient alors potentiellement utilisable en génie civil (construction routière).

\section{Interférences structurelles des $\mathbf{S H}$}

La formation de la matrice minérale se fait malgré tout en présence de forte concentration en polymères humiques. Les composés organiques, restent donc piégés dans les amas de précipitation et altèrent structurellement les produits d'hydratation (Jolicoeur 1998). La cinétique de réaction du liant est alors en interaction avec celle de la minéralisation/transformation des $\mathrm{SH}$, ce qui a d'importantes consé-
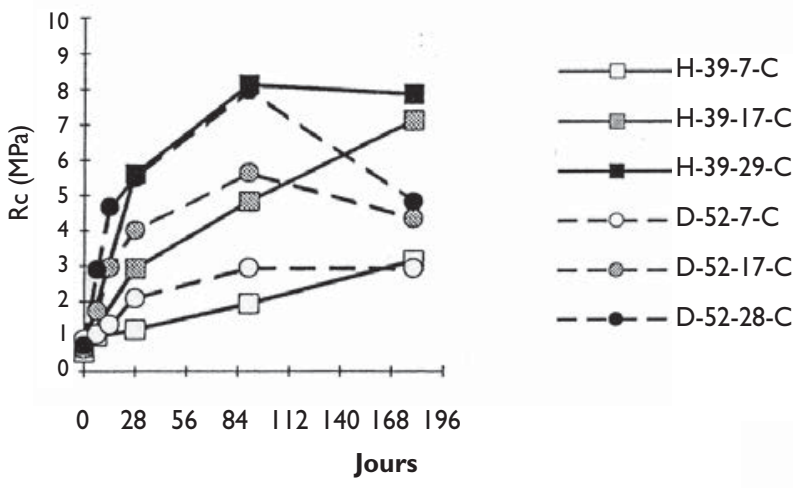

Figure 2a : Composites ciment
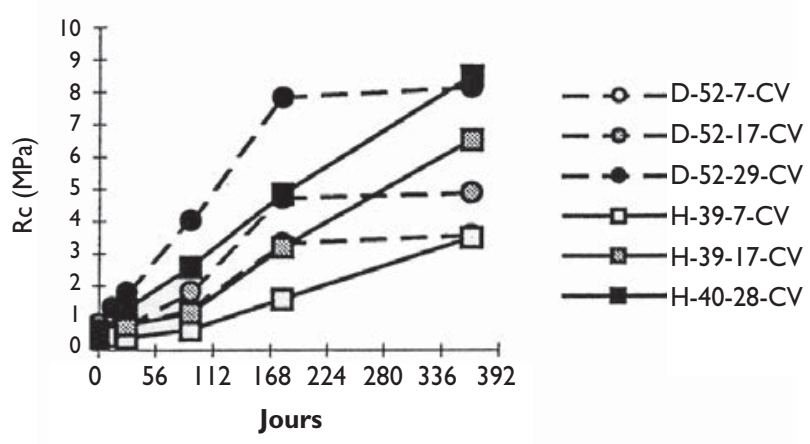

Figure 2b : Composites CV

Figure 2 : Évolution des résistances en compression simple - Influence du dosage en liant et de la cinétique de réaction quences.

La disparition des polymères humiques conduit à la disparition de l'armature polymérisée des structures minéraloorganiques et ainsi, peu à peu, à une déstructuration des agrégats organo-calciques. Dans la mesure où une structure coalescente minérale rigide les réunissait (dans le cas d'un liant à développement rapide des $\mathrm{CSH}$ ) des faiblesses internes apparaissent. Ceci est probablement la cause des chutes des caractéristiques mécaniques à long terme (voir figure 2). Dans le cas d'un développement lent des $\mathrm{CSH}$, ce mécanisme semble ne plus exister. En effet, aux membranes organiques des minéraux et aux liens organo-calciques se substituent probablement graduellement des liens $\mathrm{CSH}$ au cœur des structures floculées. La pérennité des caractéristiques mécaniques est alors assurée (voir figure 2).

Dans cette hypothèse, l'utilisation de liants et d'additifs susceptibles d'aboutir à un développement rapide des $\mathrm{CSH}$ semble donc à proscrire. L'emploi d'un liant pouzzolanique est par conséquent intéressant si l'on ne recherche pas une rigidification rapide. Une combinaison des deux types de liant semble néanmoins la plus intéressante dans le cas courant.

\section{SPÉCIFICITÉS PHYSICO-CHIMIQUES DES MATRICES ET ÉTUDE ENVIRONNEMENTALE}

\section{Développement des phases chloroaluminates}

L'étude physico-chimique révèle qu'il y a une précipitation initiale de monochloroaluminates de calcium hydratés $(\mathrm{CACH})$ et que celle-ci se poursuit probablement très longtemps dans le temps au fur et à mesure que la minéralisation/transformation des $\mathrm{SH}$ s'opère. En effet, suivant les concentrations interstitielles respectives de $\mathrm{CaCl}_{2}, \mathrm{CaSO}_{4}$, $\mathrm{H}_{2} \mathrm{CO}_{3}$, et $\mathrm{Al}_{2} \mathrm{O}_{3}$, la libération de divers éléments rend de nouveau possible la formation des chloro (sulfo, calco) aluminates de calcium hydratés. La formation de monochloroaluminates est longtemps la plus probable. Les sulfoaluminates n'ont pas été détectés par DRX et la présence de chlorures
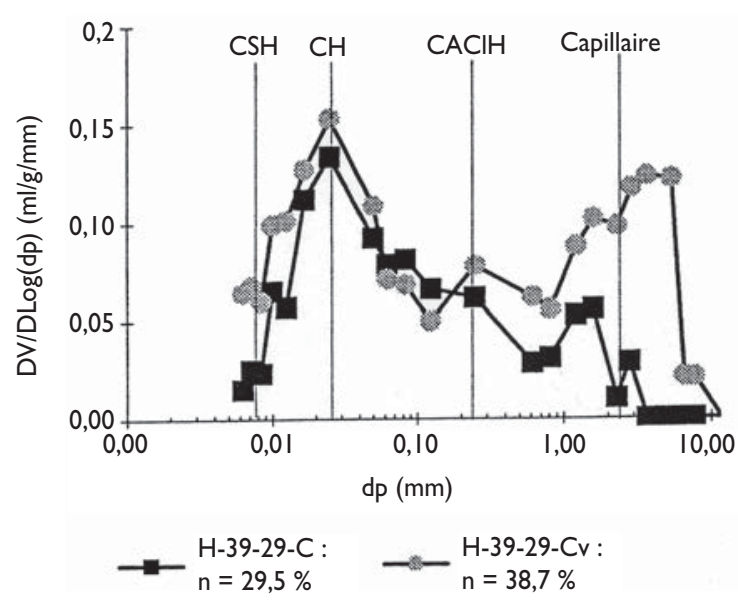

Figure 3 : Modes porosimétriques des matrices 
dans la solution interstitielle rend leur présence improbable (Capmas 1994). À long terme, après épuisement des chlorures et minéralisation des $\mathrm{SH}$, leur formation devient théoriquement de nouveau possible. Ces formations tardives sont nuisibles dans le cas où leur mode porosimétrique (principal diamètre intrinsèque des pores de la formation) serait supérieur au principal mode de la matrice déjà formée. Ceci est le cas pour les $\mathrm{CACH}$ dans les matrices $\mathrm{CH}$ (voir figure 3), ce qui entraîne de faibles gonflements à très long terme.

\section{Porosité des matrices}

En ce qui concerne la porosité intrinsèque des matrices, l'étude porosimétrique au mercure (voir figure 3) a révélé que celle-ci était trimodale et correspondait probablement à l'emboîtement des systèmes minéraux $\mathrm{CSH}-\mathrm{CH}-\mathrm{CACH}$. Cette porosité possède un volume poreux maximum pour le système $\mathrm{CH}$, ce qui est en rapport avec les dosages importants en chaux. Le système CSH est peu présent ce qui vient des faibles dosages en ajouts. Une macroporosité capillaire est présente dans le cas où la matrice ne s'effondrerait pas sur elle-même suite aux pressions de succion qu'entraîne l'auto-dessiccation. Dans le cas de l'ajout CV, ce mode porosimétrique semble provenir principalement de la désagrégation des sphérules de verre au sein de la matrice. Cette macroporosité capillaire est, par conséquent, bien plus faible dans le cas de la matrice chaux-ciment.

Pour les composites vase-chaux-CV, et par rapport au composite avec ajout de ciment, il a par ailleurs été constaté un léger décalage et un élargissement des modes porosimétriques attribués aux structures $\mathrm{CH}$ et $\mathrm{CACH}$. Ces variations, avec la porosité totale un peu plus élevée des composites CV, sont liées à l'auto-dessiccation initialement moins importante du milieu au moment du compactage. Les valeurs de porosité totale, pour un même dosage en chaux, se classent normalement dans l'ordre des dosages en ajout mais le dosage en chaux reste déterminant pour cette valeur qui est contrôlée par la baisse de la teneur en eau du matériau.

\section{Mode d'immobilisation et lixiviation des métaux}

L'importance de la porosité des matériaux va avoir une influence sur la lixiviation des polluants solubles dans la solution interstitielle par l'intermédiaire de la perméabilité. Dans le cas où la fixation chimique minérale des polluants serait peu efficace, comme cela est probablement le cas à court et moyen terme pour les métaux lourds restés complexés avec les $\mathrm{SH}$, les composites vase-chaux-CV se révéleront moins efficaces que les composites avec ajout de ciment du fait de leur porosité plus importante avec un mode capillaire également plus élevé.

Des essais de lixiviation font apparaître que pour le cuivre et le nickel, métaux sensibles à la solubilisa- tion des complexes en milieu basique (Calmano et al. 1983), cette tendance semble vérifiée. En effet, à dosages semblables, la lixiviation sur éprouvette monolithique des composites $C V$ est toujours légèrement plus importante que pour le ciment. De plus, nous constatons que l'immobilisation physique de ces métaux est peu efficace dans la mesure où la lixiviation sur monolithe est assez proche de la lixiviation sur agrégats.

Le plomb et le zinc sont par contre mieux immobilisés physiquement, comme la différence des deux modes de lixiviation le laisse penser (voir figure 4). Ces métaux semblent de plus insensibles aux différences de porosité des matrices. Ces deux éléments sont, a priori, immobilisés par les phases aluminates $(\mathrm{CAClH})$, qui sont particulièrement aptes aux substitutions avec $\mathrm{Pb}$ et $\mathrm{Zn}$ (Capmas et al. 1994). Néanmoins, on constate que la fixation chimique avec l'ajout ciment est meilleure.

Enfin, il a été établi que la lixiviation des chlorures était bien corrélée avec la porosité totale des composites (Rey 1999), ce qui suggère que la lixiviation des espèces solubles soit
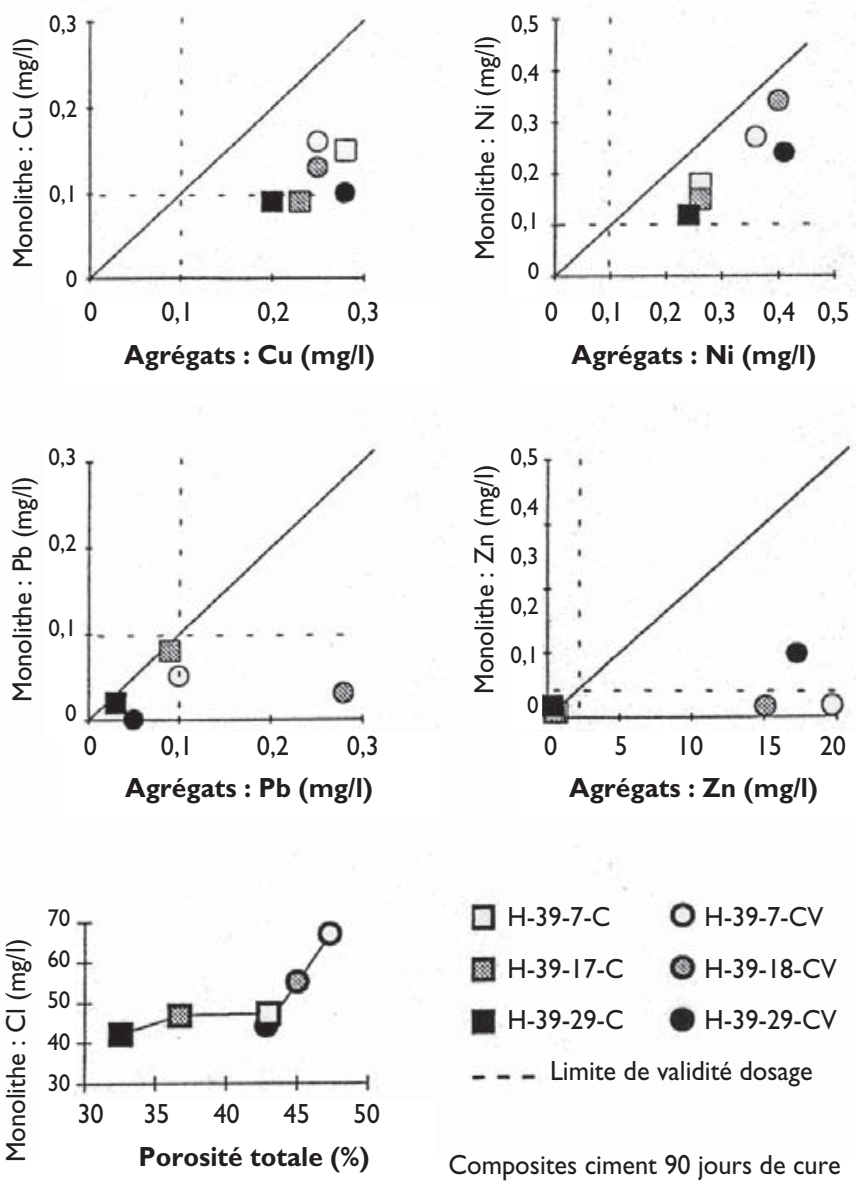

- - - Limite de validité dosage

Composites ciment 90 jours de cure

Composites CV 180 jours de cure

Lixiviation de 24 heures $(\mathrm{L} / \mathrm{S}=10)$

(NF $\times 3 \mathrm{I}-\mathrm{I} 5 \mathrm{I}$ et dosages photométriques)

Figure 4 : Résultats des lixiviations des composites et corrélation Porosité - Chlorures lixiviés 
effectivement contrôlée par celle-ci et que l'importance de la porosité capillaire (« macroporosité ») a peu d'influence sur ce phénomène.

\section{CONCLUSIONS ET PERSPECTIVES}

Nous conclurons en précisant que le traitement des vases de dragage portuaires par solidification/stabilisation à base de chaux pour constituer des composites cimentiers ou/et pouzzolaniques susceptibles de répondre à une utilisation dans le domaine du génie civil se heurte à certains facteurs comportementaux et économiques. Cependant, en tenant compte de la synthèse des conclusions phénoménologiques de cette étude, certaines possibilités s'ouvrent pour améliorer ces critères :

- d'une part l'étude d'un prétraitement des vases pour minimiser l'influence des matières organiques sur le procédé de $\mathrm{S} / \mathrm{S}$ (processus d'oxydation accéléré);

- d'autre part l'utilisation d'un liant spécifique adapté à la double problématique forte teneur en eau - matériau organique (liant pouzzolanique sulfo-alumino-calcique).

\section{* Daniel Levacher, Francis Rey,}

Upres A 6/43 Morphodynamique continentale et côtière - Groupe de recherche en génie civil - Université de Caen - 24, rue des Tilleuls - 14000 Caen

** Jean-Louis Quénec'h, Francis Rey,

École supérieure d'ingénieurs des travaux de la construction de Caen - I, rue Pierre et Marie Curie - I46I0 Epron

\section{Déchets Design}

\section{Les ambassadeurs du futur}

Gérard Bertolini

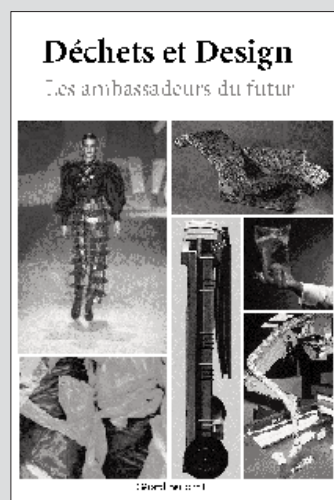

Rapprocher déchet et design peut paraître singulier. Pourtant, le déchet intervient comme mécanisme de rappel; au-delà, il interpelle les designers, et plus largement les créateurs. De plus en plus, ils devront avoir une démarche prospective, anticipatrice, préventive, remettre en cause la conception des produits pour mieux tenir compte de l'aprèsusage et des préoccupations environnementales, qui constituent des valeurs d'avenir.

Et puis, une fois n'est pas assez. Réemploi, réutilisation, recyclage, boucles et cascades, les produits doivent devenir des re-produits, les créateurs des re-créateurs.

Enfin, le produit doit être efficace dans sa mission, ainsi que dans sa démission. Pour les déchets ultimes (à réduire autant que possible), leur concours peut là encore être précieux.

Pour libérer l'avenir, le design doit investir le champ du déchet nous dit Gérard Bertolini, économiste, directeur de recherche au CNRS et spéculateur sur l'avenir des déchets.

Format $15,5^{\star} 24$ - 204 pages - $179 \mathrm{~F}$ TTC franco de port (169,67 F HT - TVA 5,5\%: 9,33 F)

Société Alpine de Publications 7, chemin de Gordes - 38100 Grenoble Tél. : 0476432864 - Fax : 0476569409

\section{Bibliographie}

-Alzieu C., 1999. Dragage et environnement marin - État des connaissances. Ed. IFREMER, $224 \mathrm{p}$.

- Boutouil M., 1998. Traitement des vases de dragage par solidification/stabilisation à base de ciment et additifs. Thèse de Doctorat, Le Havre, 258 p.

- Busnot I., 1991. Les substances humiques : complexants naturels. Thèse de Doctorat, Caen, $202 \mathrm{p}$

- Calmano W., Förstneru, Kersten M. Kraus D., 1983. Behavior of dredged mud after stabilisation with different additives. Sci. Total Environ., pp 737-747.

- Capmas A. et Sorrentino F., 1994 Étude des mécanismes de solidificationstabilisation des déchets solides par liants hydrauliques - cas des Refiom. Environnement et Technique, $\mathrm{n}^{\circ}$ I36, pp. 50-53.

- Geode, 1994. Dragage dans les ports français. Rapport de synthèse Sogreah II $3 \mathrm{p}$.

- Jolicoeur C. et Simard M.-A., 1998. Chemical admixture-cement interactions : phenomenology and physico-chemical concepts. Cement and Con. Comp. $\mathrm{n}^{\circ} 20$, pp. 87-101.

-Kamon M. et Nontananandh S., 1990.
Contribution of stainless steel slag to the development of strength for seabed hedoro. Japanese Society of Soil Mechanics and Foundation Eng., Vol. 30, n ${ }^{\circ}$ 4, pp. 63-72.

- Kujala K. et Mäkikyrö M., 1996. Effect of humus on the binding reaction in stabilized soils. Grouting and Deep Mixing, Ed. Yonekura Terashi et Shibazaki, Rotterdam, pp. 4I5-420.

- Le Roux A., 1969. Contribution à l'étude du traitement à la chaux des matériaux argileux. Thèse de Doctorat, Paris, Orsay, 105 p.

- Rey F., Levacher D., Bennabi A., Quenec'h J-L., 1998. Étude du comportement mécanique et des conditions de mise en œuvre de composites vase-chaux. $16^{\text {èmes }}$ Rencontres universitaires de génie civil, Reims, PP I22-129.

- Rey F., 1999. Étude physico-mécanique et environnementale du composite vasechaux et additifs. Thèse de Doctorat, Caen, 27I p.

-Stegemann J.A. et Cote P.L., I99I. Programme conjoint d'étude de méthodes d'essais pour l'évaluation des déchets solidifiés. Environ. Canada SPE3/HA/8, I3I p. 